\title{
Are Dosing Adjustments Required for Colchicine in the Elderly Compared with Younger Patients?
}

\author{
Suman Wason · Robert D. Faulkner · Matthew W. Davis
}

To view enhanced content go to www.advancesintherapy.com

Received: May 11, 2012 / Published online: June 29, 2012

(c) The Author(s) 2012. This article is published with open access at Springerlink.com

\section{ABSTRACT}

Introduction: The objective of this study was to compare the relative bioavailability of the US Food and Drug Administration-approved formulation of colchicine after a single $0.6 \mathrm{mg}$ dose in young (18-30 years of age) and elderly ( $\geq 60$ years of age) healthy subjects to determine whether dosing adjustments are required in elderly patients.

Methods: A single-dose, single-drug, parallelgroup study was performed in 20 young subjects with normal renal function (defined as creatinine clearance $[\mathrm{CrCl}] \geq 80 \mathrm{~mL} / \mathrm{min}$ ) and 18 elderly subjects with normal or mild renal impairment $(\mathrm{CrCl} \geq 50 \mathrm{~mL} / \mathrm{min})$ in otherwise good health. Blood samples were collected for up to 72 hours postdose and analyzed for colchicine using a

ClinicalTrials.Gov \#NCT01001052.

S. Wason $(\bowtie) \cdot$ R. D. Faulkner · M. W. Davis URL Pharma, Inc., Mutual Pharmaceutical Company, Inc., 7722 Dungan Road, Philadelphia, PA 19111, USA e-mail: swason@urlpharma.com

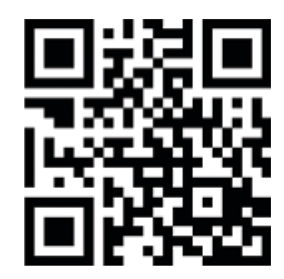

Enhanced content for Advances in Therapy articles is available on the journal web site: www.advancesintherapy.com validated liquid chromatography/tandem mass spectrometry method. Noncompartmental pharmacokinetic parameters were compared using analysis of variance methods.

Results: There were no statistically significant $(P<0.05)$ differences in mean colchicine pharmacokinetic parameters between young and elderly subjects, including peak plasma concentration $\left(\mathrm{C}_{\max }\right)(2.53$ vs. $2.56 \mathrm{ng} / \mathrm{mL})$, time to $C_{\max }(1.25$ vs. 1.25 hours), area under the plasma concentration-time curve to infinity (22.29 vs. $25.01 \mathrm{ng} / \mathrm{h} / \mathrm{mL}$ ), elimination half-life (25.4 vs. 30.1 hours), oral clearance (0.40 vs. $0.35 \mathrm{~L} / \mathrm{h} / \mathrm{kg}$ ), and apparent volume of distribution (14.3 vs. $14.8 \mathrm{~L} / \mathrm{kg}$ ), respectively.

Conclusion: The lack of any significant differences in colchicine pharmacokinetic parameters between young and elderly healthy subjects, with some of the latter including mild renal impairment, suggests that dose modification of colchicine may not be necessary in healthy elderly patients. However, when evaluating the use of colchicine dosing in an elderly patient, the confounding effect on overall exposure and safety from comorbid conditions, the use of concomitant medications, and the administration of multiple doses should be considered. 
Keywords: Age; Bioavailability; Colchicine; Dosing; Pharmacokinetics

\section{INTRODUCTION}

The prevalence of gout is increasing due to various factors, including the aging population and dietary/lifestyle changes $[1,2]$. The National Health and Nutrition Examination Survey (NHANES) estimated the prevalence of gout in the US population between 2007 and 2008 to be 8.3 million adults ( $\geq 20$ years of age), affecting 6.1 million men (5.9\% of the population) and 2.2 million women (2.0\% of the population) [3]. The overall prevalence of gout has increased from 2.7\% (NHANES III, 1988-1994) to 3.9\% (NHANES, 2007-2008) [3].

Colchicine $0.6 \mathrm{mg}$ is indicated for the prophylaxis and treatment of acute gout attacks in adults and the treatment of familial Mediterranean fever in adults and children 4 years of age and older [4]. There is limited information on the effect of age on the pharmacokinetics of colchicine.

Colchicine binds to $\beta$-tubulin heterodimers that comprise microtubules, disrupting the cytoskeleton and inducing various signaling pathways and cellular events, eventually resulting in its anti-inflammatory mechanism of action in the treatment of gout $[5,6]$. Although the exact mechanism has not been completely elucidated, colchicine inhibits the inflammasome complex in neutrophils and monocytes, interfering with the activation of the proinflammatory cytokine interleukin-1 $\beta$ [7]. The ubiquitous nature of microtubules contributes to the apparent volume of distribution $(\mathrm{Vd} / \mathrm{F})$ of colchicine, which greatly exceeds total body volume, and the slow dissociation half-life $\left(\mathrm{T}_{1 / 2}\right)$ of the tubulin-colchicine complex (20-30 hours) contributes to the prolonged plasma elimination half-life [5]. P-glycoprotein (P-gp), the adenosine triphosphate-dependent phosphoglycoprotein that is located in the cell membranes of numerous tissues, is responsible for the efflux of colchicine across membranes, including within enterocytes of the intestine. P-gp plays a predominant role in the incomplete absorption of colchicine (mean absolute bioavailability is approximately $45 \%$ ), the enterohepatic recirculation that occurs as evidenced by secondary peak plasma concentrations and for many potential drug-drug interactions (e.g., cyclosporin) $[5,6]$.

Colchicine also undergoes hepatic biotransformation by cytochrome $3 \mathrm{~A} 4$ (CYP3A4) to form three minor metabolites (2-O-demethylcolchicine, 3-O-demethylcolchicine, and 10-O-demethylcolchicine) that account for less than $5 \%$ of the parent compound in human plasma [4]. CYP3A4 inhibition of colchicine by certain drugs (e.g., macrolides, statins) has the potential to induce colchicine toxicity by dual modulation of both P-gp and CYP3A4 [6].

There is little information on the potential effect of age, gender, or race on the pharmacokinetics of colchicine. A published study investigated the pharmacokinetics of colchicine in six healthy young men and four elderly women after single-dose administration intravenously (i.v.) (0.5 mg in young men and $1 \mathrm{mg}$ in elderly women) and orally (1 mg in each group) [8]. Mean absolute bioavailability was similar in the young men and elderly women ( $44 \%$ vs. $45 \%$, respectively), whereas peak plasma concentration $\left(\mathrm{C}_{\max }\right)$ was approximately twofold higher in the elderly women compared with the young men (5.5 vs. $12 \mathrm{ng} / \mathrm{mL}$ ). Following i.v. administration, the volume of distribution at steady state $(4.2$ vs. $2.9 \mathrm{~L} / \mathrm{kg}$ ) and total body clearance (10.5 vs. $5.5 \mathrm{~L} / \mathrm{h}$ ) were reduced in the elderly women.

The pharmacokinetics of the currently approved formulation of colchicine have been 
reported in the US prescribing information [4], reviews $[5,9]$, and studies in young subjects $[10,11]$, but the potential effect of age per se on drug disposition of this formulation has not been formally investigated until now. The objective of this study was to compare the pharmacokinetics of colchicine following the oral administration of a single $0.6 \mathrm{mg}$ tablet of the approved colchicine formulation when given to young (18-30 years of age) and elderly ( $\geq 60$ years of age) healthy subjects following an overnight fast.

\section{METHODS}

\section{Subjects}

Adult male and female subjects with a body mass index of $18-30 \mathrm{~kg} / \mathrm{m}^{2}$ who were either young (18-30 years of age) or elderly ( $>60$ years of age) were eligible for recruitment if they were in good health on the basis of medical history, physical examination, and routine laboratory tests. Elderly subjects with normal renal function (defined as creatinine clearance $[\mathrm{CrCl}] \geq 80 \mathrm{~mL} / \mathrm{min}$ ) or with mild renal impairment (defined as $\mathrm{CrCl}$ between 50 and $80 \mathrm{~mL} / \mathrm{min}$ ) were allowed to participate, whereas young subjects were required to have normal renal function. The Cockcroft-Gault formula was used to measure $\mathrm{CrCl}$.

Exclusion criteria included history of allergy or sensitivity to colchicine; history of any drug hypersensitivity or intolerance likely to compromise the safety of the subject or the study in the investigator's opinion; significant history or current evidence of chronic infectious disease, system disorders, organ dysfunction (especially cardiovascular disorders), stroke, renal or hepatic disorder, diabetes, or bleeding disorders; presence of gastrointestinal disease or history of malabsorption in the previous year; presence of any medical condition requiring regular treatment or prescription drug treatment; history of psychiatric disorders within the previous 2 years that required hospitalization or medication; use of pharmacologic agents known to induce or inhibit drug-metabolizing enzymes (especially CYP3A4) within 30 days before dosing; use of a study drug in a research investigation within 30 days before dosing; history of treatment for drug or alcohol addiction, or excessive alcohol consumption (>14 units/week on average) within the previous year; positive test result for drug abuse; use of tobacco products within 90 days before dosing; positive HIV, hepatitis B surface antigen, or hepatitis $\mathrm{C}$ antibody test; difficulty in fasting or eating standard meals; donation or significant loss of whole blood ( $\geq 480 \mathrm{~mL}$ ) within 30 days or plasma within 14 days before dosing; inability or unwillingness to tolerate multiple venipuncture; and women with a positive serum pregnancy test result, who were likely to become pregnant during the study, or who were lactating. Furthermore, women of childbearing potential had to be prepared to abstain from sexual intercourse or have used and continue to use a reliable method of contraception (e.g., use of condom with spermicide, intrauterine device, hormonal contraception) 30 days before dosing and throughout the study.

\section{Study Design}

The study protocol received ethics committee approval (Novum Independent Institutional Review Board). All subjects provided written informed consent before study participation, which was conducted in accordance with the US Code of Federal Regulations and International Conference on Harmonisation Guidelines for Good Clinical Practice, and adhered to the ethical principles of the Declaration of Helsinki. The study was performed at a single study center (Novum Pharmaceutical Research Services, Las Vegas, Nevada, USA). 
Following a screening period of up to 4 weeks, all subjects received a single $0.6 \mathrm{mg}$ colchicine tablet administered with $240 \mathrm{~mL}$ of water at room temperature. They were instructed to swallow the tablet whole without chewing or biting. Dosing order was randomly assigned by age group in blocks of two.

All subjects checked into the clinical investigation facility on day 1 with an evening meal served and consumed more than 10 hours before dosing. No food or beverages (except water) were permitted until 4 hours after studydrug administration on day 1 . Standardized meals and snacks were served at approximately 4, 9, and 13 hours after dosing during confinement in the test facility. Blood sample collections were performed before meals if sampling and meal times coincided. No caffeine, xanthine, alcohol, or grapefruit products were permitted during confinement; subjects were also instructed to abstain from any food or beverages containing these products within 48 hours before dosing and throughout the period of blood sample collection. During the confinement period of the study, additional fluids were not permitted from 1 hour before to 1 hour after dosing except for the water administered with the test dose. Otherwise, water was freely encouraged, although no fluids other than water or those served with standardized meals were permitted. Subjects left the clinical facility approximately 24 hours after test dose administration (day 2) but returned to provide 36 , 48, 60, and 72-hour blood sample collections.

Subjects were not permitted to take prescription medications except for hormonal contraception within 2 weeks before dosing and over-the-counter medications including vitamins and herbal products within 3 days before dosing and throughout the duration of blood sample collection.

Subjects were advised that they could withdraw from the study at any time for any reason.
Furthermore, the investigator could withdraw subjects from the study to protect their health or for noncompliance with study procedures. Any subject experiencing vomiting within 3 hours after dosing (based on twice the expected time to $\mathrm{C}_{\text {max }}\left[\mathrm{T}_{\text {max }}\right]$ of $\sim 1.5$ hours) would be dropped from analysis. No subjects were discontinued because of these criteria.

Medical history, physical examination, 12-lead electrocardiography, routine laboratory tests, drug screen, virology tests for HIV and hepatitis viruses, and serum pregnancy test were performed during the 4-week screening period. Vital signs were measured during the screening period and on days $-1,1$, and 2, with regular testing on day 1 before dosing and at regular intervals after dosing. Urine pregnancy test and repeat drug screen were performed on admission to the clinical facility on day -1 . Medical history was reviewed again on day 2 , on days $3-4$, and on discharge from the clinical facility. Routine laboratory tests were also repeated at discharge from the clinical facility. Any undesirable sign, symptom, or medical condition occurring after starting the study, whether reported spontaneously, in response to questioning, or directly observed, was recorded regardless of suspected relation to the study medication. All treatment-emergent adverse events (AEs) were recorded (coded using MedDRA version 12.0 adverse event dictionary) and were graded by intensity (mild, moderate, or severe) and relationship to the study drug (unlikely, possible, or probable) by the investigator.

\section{Pharmacokinetic Measurements}

Venous blood samples (6 $\mathrm{mL}$ in prechilled EDTA tubes) were taken by direct venipuncture at 0 (predose), 0.5, 1, 1.5, 2, 2.5, 3, 4, 5, 6, 7, 8, 9, $10,11,12,16,18,24,36,48,60$, and 72 hours postdose. Samples were mixed by gently inverting 
the tubes several times and centrifuged at approximately 2,700-3,000 rpm for 10 minutes at $4^{\circ} \mathrm{C}$. Plasma samples were collected into polypropylene tubes and stored frozen to at least $-18^{\circ} \mathrm{C}$ until analysis. The time from blood collection to being centrifuged was less than 60 minutes and to plasma being frozen was less than 120 minutes. Frozen plasma samples on dry ice were transported overnight for assay at a single analytical laboratory (Frontage Laboratories, Inc, Malvern, Pennsylvania, USA) using a liquid chromatography/tandem mass spectrometry (LC/ MS/MS) method. For assay, $100 \mu \mathrm{L}$ of thawed plasma was mixed with $20 \mu \mathrm{L}$ of diluent 1 (methanol/water, 50:50), $20 \mu \mathrm{L}$ of internal standard (colchicine- $\mathrm{d}_{3} 5 \mathrm{ng} / \mathrm{mL}$ ), and $300 \mu \mathrm{L}$ of diluent 2 (ammonium formate $400 \mathrm{nmol} / \mathrm{L}$ in water). Then, $1.5 \mathrm{~mL}$ of extraction solvent (methyl tert-butyl ether/ethyl acetate, 60:40) was added, mixed for 10 minutes, and centrifuged for 5 minutes at $3,000 \mathrm{rpm}$. The organic layer was removed, dried at $35^{\circ} \mathrm{C}$ under nitrogen using an evaporator for approximately 20 minutes, and reconstituted in $200 \mu \mathrm{L}$ of mobile phase (methanol/ethyl acetate, 60:40, with ammonium formate $2 \mathrm{mmol} / \mathrm{L}$ ). A $20-\mu \mathrm{L}$ aliquot was injected (Shimadzu liquid chromatography pump and autosampler) onto LC/MS/MS (Sciex API 5000). A Synergi Polar-RP, $50 \times 2.0 \mathrm{~mm}, 4-\mu \mathrm{m}$ column was used with a pump flow rate of $0.6 \mathrm{~mL} / \mathrm{min}$. Analysis was performed in positive ionization mode, with colchicine and internal standard identified by the multiple reaction monitoring transitions $\mathrm{m} / \mathrm{z} 400.2 \rightarrow 310.1$ and $\mathrm{m} / \mathrm{z} 402.1 \rightarrow 310.1$, respectively. The assay calibration range was $0.02-20 \mathrm{ng} / \mathrm{mL}$ for analytical runs with a lower limit of quantitation of $0.02 \mathrm{ng} / \mathrm{mL}$. Intraday accuracy was $99.1-110.0 \%$ with intraday precision of 2.35-17.54 coefficient of variation $(\% \mathrm{CV})$, and interbatch accuracy was 99.17-100.05\% (1.92-13.32\%CV).

Model-independent pharmacokinetic parameters for colchicine were determined using the SAS (version 9.1.3 or later), including $\mathrm{C}_{\max } ; \mathrm{T}_{\max } ; \mathrm{AUC}_{0-\mathrm{t}}$ (area under the plasma concentration-time curve [AUC] from time zero to time $t$, where $t$ is the time of the last measurable concentration $\left[\mathrm{C}_{\mathrm{t}}\right]$ calculated using the linear trapezoidal method); $\mathrm{AUC}_{0-\infty}$ (AUC from time zero extrapolated to infinity calculated as $\mathrm{AUC}_{0-\mathrm{t}}+\mathrm{C}_{\mathrm{t}} / \mathrm{K}_{\mathrm{el}}$, where $\mathrm{C}_{\mathrm{t}}$ is the last measurable drug concentration and $\mathrm{K}_{\mathrm{el}}$ is the elimination rate constant estimated via linear regression of the terminal portion of the log concentration versus time curve); $\mathrm{T}_{1 / 2}$ (elimination half-life calculated as $\ln [2] / \mathrm{K}_{\mathrm{el}}$ ); CL/F (apparent clearance calculated as the dose/AUC ${ }_{0-t}$ ); and $\mathrm{Vd} / \mathrm{F}$ (apparent volume of distribution, calculated as $\mathrm{CL} / \mathrm{K}_{\mathrm{el}}$ ).

\section{Statistical Analysis}

Descriptive statistics (mean $\pm \mathrm{SD}$ ) were used to summarize the pharmacokinetic data for each age group. Analyses of variance (ANOVA) were performed using the general linear model procedure of SAS (version 9.1.3 or later) with hypothesis testing for study-drug effects at $\alpha=0.05$. The statistical model contained the main effect of group. Least square means for the groups (LSMEANS statement), the differences between adjusted group means, and the standard errors associated with these differences (ESTIMATE statement) were calculated. Comparison of $\mathrm{C}_{\max }, \mathrm{AUC}_{0-\mathrm{t}}$, and $\mathrm{AUC}_{0-\infty} 90 \%$ confidence intervals (CI) for each group was constructed to test two one-sided hypotheses at the $\alpha=0.05$ level of significance, with confidence intervals presented for the geometric mean ratios obtained from logarithmic (ln)transformed data. In addition, the relationship between colchicine pharmacokinetic parameters and covariates including age and $\mathrm{CrCl}$ were investigated by linear regression analysis. Post-hoc analysis of differences in race and gender $(n=38)$ was also performed. 


\section{RESULTS}

Baseline demographic and clinical characteristics for the 38 subjects (20 young and 18 elderly; 20 women and 18 men; 14 African American, 15 white, and 9 "other") included in the statistical analysis are summarized in Table 1. Elderly subjects in this study had a median age of 62 years and a mean age of $62.8 \pm 2.83$ years.
No subjects older than 70 years were enrolled in the study. The age groups were balanced for weight and body mass index. There were significant differences in mean age $(P<0.001)$ and $\mathrm{CrCl}(P<0.001)$ between the young and elderly groups. There was a statistically significant difference $(P<0.001)$ in $\mathrm{CrCl}$ between age groups $(132 \pm 23.2 \mathrm{~mL} / \mathrm{min}$ for young vs. $87.0 \pm 17.9 \mathrm{~mL} / \mathrm{min}$ for elderly subjects).

Table 1 Baseline demographic and clinical characteristics

\begin{tabular}{|c|c|c|}
\hline Characteristic & $\begin{array}{l}\text { Young subjects } \\
\qquad(n=20)\end{array}$ & $\begin{array}{l}\text { Elderly subjects } \\
\qquad(n=18)\end{array}$ \\
\hline \multicolumn{3}{|l|}{ Gender, $n(\%)$} \\
\hline Male & $8(40.0)$ & $10(55.6)$ \\
\hline Female & $12(60.0)$ & $8(44.4)$ \\
\hline \multicolumn{3}{|l|}{ Ethnicity, $n(\%)$} \\
\hline Hispanic & $4(20.0)$ & $3(16.7)$ \\
\hline Non-Hispanic & $16(80.0)$ & $15(83.3)$ \\
\hline \multicolumn{3}{|l|}{ Race, $n(\%)$} \\
\hline American Indian or Alaska Native & 0 & $2(11.1)$ \\
\hline Black or African American & $13(65.0)$ & $1(5.6)$ \\
\hline White & $3(15.0)$ & $12(66.7)$ \\
\hline Other & $4(23.5)$ & $3(16.7)$ \\
\hline Median age, year (range) & $25(18-30)$ & $62(60-70)$ \\
\hline Mean age, year $\pm S D$ & $24.41 \pm 3.66^{*}$ & $62.83 \pm 2.83^{*}$ \\
\hline Median height, cm (range) & $172.2(154.9-188.0)$ & $167.6(152.4-180.3)$ \\
\hline Mean height, $\mathrm{cm} \pm \mathrm{SD}$ & $67.29 \pm 3.62$ & $65.50 \pm 3.42$ \\
\hline Median weight, $\mathrm{kg}$ (range) & $80.3(52.6-95.3)$ & $71.2(61.2-92.5)$ \\
\hline Mean weight, $\mathrm{kg} \pm \mathrm{SD}$ & $170.53 \pm 26.20$ & $162.94 \pm 21.69$ \\
\hline Median body mass index, $\mathrm{kg} / \mathrm{m}^{2}$ (range) & $25.8(20.4-29.9)$ & $27.6(20.4-30.0)$ \\
\hline Mean body mass index, $\mathrm{kg} / \mathrm{m}^{2} \pm \mathrm{SD}$ & $26.19 \pm 2.81$ & $26.54 \pm 3.19$ \\
\hline Median $\mathrm{CrCl}, \mathrm{mL} / \mathrm{min}$ (range) & $123(83-183)^{*}$ & $86.0(57-120)^{*}$ \\
\hline Mean $\mathrm{CrCl}, \mathrm{mL} / \mathrm{min} \pm \mathrm{SD}$ & $132.56 \pm 23.16^{*}$ & $87.02 \pm 17.92^{*}$ \\
\hline
\end{tabular}


Race was predominantly black among the young subjects and white among the elderly subjects, with a significant between-group difference $(P<0.001)$. All 38 subjects were evaluated for safety.

Mean plasma concentration-time profiles for colchicine in the young and elderly subjects are graphically presented in Fig. 1. The arithmetic mean pharmacokinetic parameters for colchicine in the young and elderly groups are summarized in Table 2. Statistical comparisons revealed no statistically significant $(P<0.05)$ differences between the age groups for any of the colchicine pharmacokinetic parameters.

The relationship between exposure to colchicine (AUC) and $\mathrm{CrCl}$ is presented graphically in Fig. 2 . A trend for higher colchicine systemic exposure with decreasing renal function (as measured by $\mathrm{CrCl}$ ) was seen; however, the magnitude of the differences was small and not clinically meaningful. With regard to gender, there was a significant difference $(P<0.05)$ in AUCs; however, the mean ratio for women to men was 1.21 , a difference deemed not clinically meaningful. The mean $\mathrm{C}_{\max }$ for colchicine was similar in the young and elderly groups ( 2.61 vs. $2.56 \mathrm{ng} / \mathrm{mL}$, respectively).

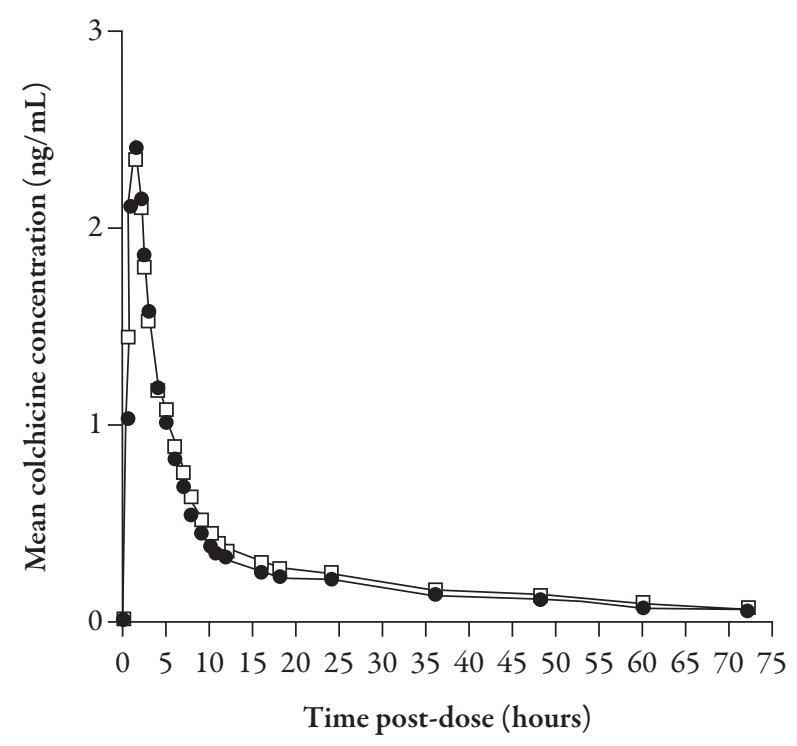

Fig. 1 Mean plasma colchicine concentrations following oral administration of a single $0.6 \mathrm{mg}$ colchicine tablet to young $(\bullet)$ and elderly $(\square)$ subjects

Table 2 Arithmetic mean pharmacokinetic parameters by age group for colchicine administered as a single 0.6-mg tablet to healthy subjects $(n=38)$

\begin{tabular}{|c|c|c|c|}
\hline \multirow[t]{2}{*}{ Parameter } & \multicolumn{2}{|c|}{ Arithmetic mean $\pm \mathrm{SD}(\% \mathrm{CV})$} & \multirow{2}{*}{$\begin{array}{l}P \text { value for between-group } \\
\text { comparison }\end{array}$} \\
\hline & $\begin{array}{l}\text { Young } \\
(n=20)\end{array}$ & $\begin{array}{l}\text { Elderly } \\
(n=18)\end{array}$ & \\
\hline $\mathrm{AUC}_{0-\mathrm{t}}(\mathrm{ng} / \mathrm{h} / \mathrm{mL})$ & $19.95 \pm 5.86(29.4)$ & $21.88 \pm 6.22(28.4)$ & 0.3311 \\
\hline $\mathrm{AUC}_{0-\infty}(\mathrm{ng} / \mathrm{h} / \mathrm{mL})$ & $22.39 \pm 6.95(31.3)$ & $25.01 \pm 6.92(27.7)$ & 0.2352 \\
\hline $\mathrm{C}_{\max }(\mathrm{ng} / \mathrm{mL})$ & $2.61 \pm 0.71(27.6)$ & $2.56 \pm 0.97(38.0)$ & 0.9237 \\
\hline $\mathrm{T}_{\max }(\mathrm{h})$ & $1.38 \pm 0.42(39.6)$ & $1.25 \pm 0.43(34.3)$ & 0.9869 \\
\hline $\mathrm{K}_{\mathrm{el}}\left(\mathrm{h}^{-1}\right)$ & $0.028 \pm 0.006(20.3)$ & $0.025 \pm 0.01(28.7)$ & 0.1459 \\
\hline $\mathrm{T}_{1 / 2}(\mathrm{~h})$ & $24.9 \pm 5.34(20.6)$ & $30.06 \pm 10.78(35.9)$ & 0.0936 \\
\hline $\mathrm{CL} / \mathrm{F}(\mathrm{L} / \mathrm{h} / \mathrm{kg})$ & $0.400 \pm 0.121(30.1)$ & $0.351 \pm 0.10(29.0)$ & 0.1876 \\
\hline $\mathrm{Vd} / \mathrm{F}(\mathrm{L} / \mathrm{kg})$ & $14.3 \pm 4.31(30.0)$ & $14.8 \pm 5.59(37.8)$ & 0.7854 \\
\hline
\end{tabular}

$A U C_{0-\mathrm{t}}$ area under the plasma concentration-time curve to the last measurable time point, $A U C_{0-\infty}$ area under the plasma concentration-time curve to time infinity, $C_{\max }$ peak plasma concentration, $\% C V$ coefficient of variation, $T_{\max }$ time to reach $\mathrm{C}_{\max ,} K_{\mathrm{el}}$ elimination rate constant, $t_{1 / 2}$ terminal half-life, $C L / F$ apparent clearance, $V d / F$ apparent volume of distribution 


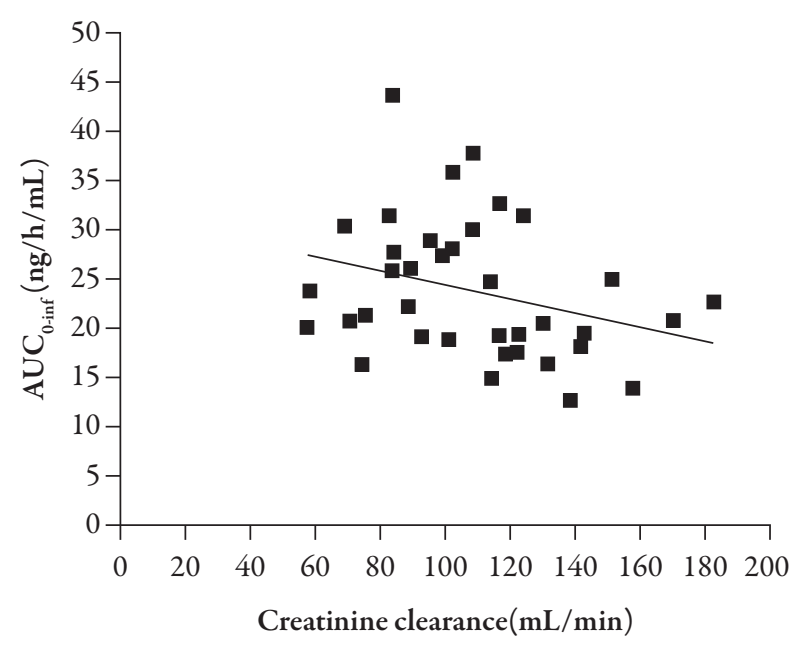

Fig. 2 Colchicine exposure (AUC) versus creatinine clearance. $A U C$ area under the plasma concentration-time curve
The $\mathrm{C}_{\max }$ ratio (elderly/young) for colchicine was close to unity (0.989 [90\% CI 0.81-1.16]). The $\mathrm{AUC}_{0-\mathrm{t}}$ and $\mathrm{AUC}_{0-\infty}$ ratios for colchicine were slightly less than unity $(0.91$ [90\% CI 0.76-1.06] and 0.89 [90\% CI 0.74-1.04], respectively), indicating marginally higher exposure to colchicine in the elderly patients, although the difference was not significant $(P>0.05)$. The mean elimination $\mathrm{T}_{1 / 2}$ was slightly longer in subjects aged 60 years and older than in those 18-30 years of age; however, this was not significant $(P>0.05)$. Furthermore, there were no differences in oral clearance $(\mathrm{CL} / \mathrm{F}$; $P>0.05)$ or apparent volume of distribution $(\mathrm{Vd} / \mathrm{F}$; $P=0.7854$ ) between age groups.

Table 3 Study-drug emergent adverse events $(n=38)$

\begin{tabular}{lcc}
\hline AE & \multicolumn{2}{c}{ Subjects, $n$ (\%) } \\
\cline { 2 - 3 } & Young & Elderly \\
& $(n=20)$ & $(n=18)$ \\
\hline Any AE & $7(35.0)$ & $8(44.4)$ \\
AE by preferred term $^{\mathrm{a}}$ & & $7(38.9)$ \\
Increased BP $^{\mathrm{b}}$ & $1(5.0)$ & $2(11.1)$ \\
Somnolence $^{\mathrm{c}}$ & $1(5.0)$ & 0 \\
Headache $^{\mathrm{c}}$ & $2(10.0)$ & $1(5.6)$ \\
Tinnitus $^{\mathrm{c}}$ & 0 & 0 \\
Abdominal discomfort $^{\mathrm{c}}$ & $1(5.0)$ & 0 \\
Abdominal pain $^{\left(\text {upper }^{\mathrm{c}}\right.}$ & $1(5.0)$ & 0 \\
Nausea $^{\mathrm{c}}$ & $1(5.0)$ & $1(5.6)$ \\
Feeling hot $^{\mathrm{c}}$ & 0 & 0 \\
BP decreased $^{\mathrm{b}}$ & $1(5.0)$ & 0 \\
Heart rate increased $^{\mathrm{b}}$ & $1(5.0)$ & $1(5.6)$ \\
Dizziness $^{\mathrm{c}}$ & 0 & 0 \\
Nasal congestion $^{\mathrm{b}}$ & $1(5.0)$ & \\
\hline AE & & 0 \\
\hline
\end{tabular}

$A E$ adverse event, $B P$ blood pressure

a According to MedDRA version 12.0

${ }^{\mathrm{b}}$ Relationship to drug unlikely

${ }^{\mathrm{c}}$ Relationship to drug possible 
ANOVA comparisons by gender showed slightly higher $C_{\max }(2.76$ vs. $2.38 \mathrm{ng} / \mathrm{mL})$ and AUC (26.2 vs. $21.7 \mathrm{ng} / \mathrm{h} / \mathrm{mL}$ ) in female subjects, differences that were not statistically significant $(P>0.05)$. Furthermore, no significant gender differences were seen in $\mathrm{T}_{\max }$, AUC, CL/F, Vd/F, or $T_{1 / 2}$. There were no significant differences in colchicine pharmacokinetic parameters between the racial groups.

A total of 15 of the 38 subjects reported 22 treatment-emergent AEs, with seven in the young group and eight in the elderly group (Table 3 ). None of the AEs was considered serious. All 22 AEs were classified as mild in intensity and resolved spontaneously before study completion. The AE relationship to drug was considered unlikely $(n=11)$ or possible $(n=11)$. The most frequent AEs were increased blood pressure $(n=8)$ and somnolence $(n=3)$. The occurrence of gastrointestinal disturbances related to therapy with multiple doses of colchicine is typically higher $(26 \%$ in a clinical trial of patients with acute gout flare) [4] than was reported in this single-dose study of colchicine (5\%). This same clinical study does not, however, account for the rate of increased blood pressures observed in the present study ( $5 \%$ young subjects $[n=1]$ and $38.9 \%$ elderly subjects $[n=7]$ ) [4]. For the occurrence of increased blood pressure, it was determined that a relationship to study drug in this study was unlikely.

\section{DISCUSSION}

The study sought to compare the relative bioavailability of the US Food and Drug Administration (FDA)-approved formulation of colchicine after a single $0.6 \mathrm{mg}$ dose in young (18-30 years of age) and elderly healthy subjects ( $\geq 60$ years of age) to determine whether dosing adjustments are required in elderly patients. There were two identifiable, potential limitations to this study. First, the age of the elderly population was limited to a range of 60-70 years to avoid potential confounding issues of renal dysfunction. Second, the study evaluated only healthy subjects without comorbid conditions, such as gout, because FDA guidance on drug-drug interaction studies indicate that it is reasonable to study healthy volunteers. In addition, elderly patients with mild renal impairment were allowed to participate at the discretion of the investigator because they are generally considered to be healthy and adjustment of dosing is not required for treatment of gout flare, prophylaxis of gout flare, and familial Mediterranean fever. However, patients should be monitored closely [4].

Single-dose colchicine $0.6 \mathrm{mg}$ was well tolerated in young and elderly healthy subjects. There were no clinically meaningful differences in colchicine pharmacokinetic parameters (i.e., $\mathrm{C}_{\max }$, AUC, $\mathrm{T}_{1 / 2}$, and $\mathrm{CL} / \mathrm{F}$ ) between young and elderly healthy subjects, including those with mild renal impairment. In addition, there were no statistically significant differences $(P<0.05)$ observed in the comparisons of the pharmacokinetic parameters by race or gender. Although the study was not designed to examine gender or race differences in colchicine pharmacokinetics, comparisons were performed on the pooled data across the two age groups by gender and race.

\section{CONCLUSION}

The only previous recommendation for colchicine dosing was reported by Terkeltaub [12], who suggested that the recommended maintenance dose of colchicine should be reduced by half in patients 70 years of age or older. These data were empirical and not based on actual study results.

In this study, the lack of any clinically meaningful differences in colchicine pharmacokinetic parameters between healthy 
young and elderly subjects, with some of the latter having mild renal impairment, suggests that dose modification of colchicine according to age may not be necessary in adults. These findings require confirmation in patients with gout and, in general, dose selection for colchicine in elderly patients with gout should be performed with caution, taking into consideration the presence of comorbid conditions, concomitant medications, and multiple doses.

\section{ACKNOWLEDGMENTS}

This is a PRISM publication. Mutual Pharmaceutical Company's PRISM (PRogram to Improve the Safety of Medication) research initiative provides for improvement of a product's safety profile and lifecycle management by focusing on metabolic pathways, thereby identifying methods for optimizing safety and patient outcomes regardless of drug class. PRISM discoveries provide valuable information to the physician, pharmacist, and patient, and are incorporated into the product label as required by FDA guidance.

Dr. Wason is the guarantor for this article, and takes responsibility for the integrity of the work as a whole.

Medical editorial assistance was provided by Peter Todd, PhD, and James A. Shiffer, RPh, CCP, Write On Time Medical Communications, LLC, and was funded by Mutual Pharmaceutical Company, Inc., a wholly owned subsidiary of URL Pharma, Inc. The authors acknowledge Darin B. Brimhall, DO, CPI, from Novum Pharmaceutical Research Services, located in Las Vegas, NV, for his contribution as the principal investigator for this study. The authors would also like to acknowledge study leader and project manager Kimberly Stulir, MBA, Robert Faulkner, PhD, Thomas Lauterio, PhD, and Deborah
DeMaria, MS (Mutual Pharmaceutical Company, Inc.) for their review and critical revisions for important intellectual content.

Conflict of Interest. S.W. is employed by Mutual Pharmaceutical Company, Inc., a wholly owned subsidiary of URL Pharma, Inc., and holds employee equity shares in the parent company. R.D.F. is employed by Mutual Pharmaceutical Company, Inc., a wholly owned subsidiary of URL Pharma Inc., and holds employee equity shares in the parent company. M.W.D. is employed by Mutual Pharmaceutical Company, Inc., a wholly owned subsidiary of URL Pharma, Inc., and holds employee equity shares in the parent company as well as 13 patents pertaining to colchicine.

Funding Statement. Supported by Mutual Pharmaceutical Company, Inc., a wholly owned subsidiary of URL Pharma, Inc.

Open Access. This article is distributed under the terms of the Creative Commons Attribution Noncommercial License, which permits any noncommercial use, distribution, and reproduction in any medium, provided the original author(s) and source are credited.

\section{REFERENCES}

1. Choi HK, Mount DB, Reginato AM. Pathogenesis of gout. Ann Intern Med. 2005;143:499-516.

2. Roddy E, Zhang W, Doherty M. The changing epidemiology of gout. Nat Clin Pract Rheumatol. 2007;3:443-9.

3. Zhu Y, Pandya BJ, Choi HK. Prevalence of gout and hyperuricemia in the US general population: the National Health and Nutrition Examination Survey 2007-2008. Arthritis Rheum. 2011;63:3136-41.

4. Colcrys ${ }^{\circledR}$ (colchicine, USP) tablets for oral use: prescribing information, 14 March 2012. Available at: www.colcrys.com/assets/pdf/COLCRYS_Full_ Prescribing_Information.pdf. Accessed Apr 252012.

5. Niel E, Schermann J-M. Colchicine today. Joint Bone Spine. 2006;73:672-8. 
6. Terkeltaub RA. Colchicine update: 2008. Semin Arthritis Rheum. 2009;38:411-19.

7. Martinon F, Pétrilli V, Mayor A, Tardivel A, Tschopp J. Gout-associated uric acid crystals activate the NALP3 inflammasome. Nature. 2006;440:237-41.

8. Rochdi M, Sabouraud A, Girre C, Venet R, Scherrmann JM. Pharmacokinetics and absolute bioavailability of colchicine after i.v. and oral administration in healthy human volunteers and elderly subjects. Eur J Clin Pharmacol. 1994;46:351-4.

9. Nuki G. Colchicine: its mechanism of action and efficacy in crystal-induced inflammation. Curr Rheumatol Rep. 2008;10:218-27.
10. Chappey ON, Niel E, Wautier JL, et al. Colchicine disposition in human leukocytes after single and multiple oral administration. Clin Pharmacol Ther. 1993;54:360-7.

11. Terkeltaub RA, Furst DE, Bennett K, Kook KA, Crockett RS, Davis MW. High versus low dosing of oral colchicine for early acute gout flare: twenty-four-hour outcome of the first multicenter, randomized, double-blind, placebo-controlled, parallel-group, dosecomparison colchicine study. Arthritis Rheum. 2010;62:1060-8.

12. Terkeltaub RA. Gout. $\mathrm{N}$ Engl J Med. 2003;349:1647-55. 